PROCEEDINGS OF THE

AMERICAN MATHEMATICAL SOCIETY

Volume 139, Number 1, January 2011, Pages 185-199

S 0002-9939(2010)10457-4

Article electronically published on July 2, 2010

\title{
JOINING PRIMENESS AND DISJOINTNESS FROM INFINITELY DIVISIBLE SYSTEMS
}

\author{
MARIUSZ LEMAŃCZYK, FRANÇOIS PARREAU, AND EMMANUEL ROY \\ (Communicated by Bryna Kra)
}

\begin{abstract}
We show that ergodic dynamical systems generated by infinitely divisible stationary processes are disjoint in the sense of Furstenberg from distally simple systems and systems whose maximal spectral type is singular with respect to the convolution of any two continuous measures.
\end{abstract}

\section{INTRODUCTION}

The notion of disjointness of dynamical systems introduced by Furstenberg 9 in 1967 leads to a natural program of identifying disjoint classes of dynamical systems. We refer to the recent monograph [11] and also to [34 for the joining theory of dynamical systems. In this note we introduce the notion of a joining prime (JP) system. Such a system $T$ has the property that every ergodic joining $\lambda$ of $T$ with the Cartesian product $S_{1} \times \ldots \times S_{k}$ of weakly mixing systems is the direct product of a joining of $T$ and some $S_{i}$ with the remaining factors $S_{j}$. In particular, a weakly mixing JP system does not admit a Cartesian product representation, and it is natural to expect that it will be disjoint from classes of systems which are sufficiently divisible in some sense. Indeed, we will show that the JP class is disjoint from a class of systems which includes systems of probabilistic origin, namely those arising from infinitely divisible (ID) stationary processes, and also from infinitely divisible systems in the ergodic theoretical sense (to avoid confusion, we will refer to the latter as ergodically ID systems).

The JP class includes two recently considered classes of automorphisms: (A) some generalizations of simple systems (2-quasi-simple systems), considered by Ryzhikov and Thouvenot in [33, and later in [16] (distally simple systems); (B) systems whose maximal spectral type is singular with respect to the convolution of any two continuous measures on the circle.

Simple systems were introduced by Veech [36] and del Junco and Rudolph [18. We recall here that this class includes some horocycle flows [27, and in fact all

Received by the editors October 28, 2009 and, in revised form, February 22, 2010.

2010 Mathematics Subject Classification. Primary 37A05; Secondary 37A10, 37A30, 37 A50.

Key words and phrases. Joinings, disjointness, infinite divisibility, distal simplicity, spectral singularity and convolutions.

The research of the first author is partially supported by Polish MNiSzW grant N N201 384834, Marie Curie "Transfer of Knowledge" EU program, project MTKD-CT-2005-030042 (TODEQ).

The research of the first and third authors is partially supported by the MSRI (Berkeley) program "Ergodic Theory and Additive Combinatorics".

(C)2010 American Mathematical Society Reverts to public domain 28 years from publication 
horocycle flows are factors of simple systems [34. Another subclass of simple systems comes from finite rank theory (see [17, [19]). In [15], simple systems were shown to be disjoint from Gaussian systems; earlier, in [34, Thouvenot showed disjointness of Gaussian systems from systems with the minimal self-joining property. This disjointness result was generalized in two directions. In 34, Thouvenot introduced ergodically ID systems, extending some properties of Gaussian systems: an ergodically ID system possesses an infinite tree of splitting factors, in which for every path the intersection of the decreasing family of factors is trivial.

Moreover, a larger class of systems was recently considered in [33], namely those systems for which all ergodic self-joinings different from the product measure are relatively isometric over the marginal factors. The disjointness of such systems from some subclasses of ergodically ID systems including Gaussian systems was then proved in [33. and 35]. If we replace relatively isometric by relatively distal and we assume additionally the so-called PID property (each pairwise independent self-joining of such a system is independent), then we get the class of so-called distally simple systems, which are shown to be disjoint with all ergodically ID systems in [16]. All flows with the Ratner property [27] are distally simple. Also, partially mixing finite rank transformations lie in the class of distally simple systems 22], 34. Moreover, recently in [8, it is proved that some smooth flows on surfaces satisfy the Ratner property and hence are distally simple.

On the other hand, the JP class includes those systems whose maximal spectral type is singular with respect to the convolution of any two continuous measures, a property which we refer to as the $C S$ property. A stronger property is that the Gaussian automorphism constructed from the (reduced) maximal spectral type has a simple spectrum (simple convolutions property). The famous Chacon automorphism has both the minimal self-joinings property [17] and the simple convolution property [1]. Ageev [2] and Ryzhikov [32] recently showed that many other rank one transformations enjoy the simple convolution property, including typical automorphisms and mixing examples. These properties are also related to the property that the convolution powers of the (reduced) maximal spectral type are mutually singular (see [20, and also [14] and 34] for the usefulness of such a property in ergodic theory).

The extension of the definitions and results to flows and actions of other locally compact abelian groups is straightforward. In Section 6, we will show that when $\mathcal{T}=\left(T_{t}\right)_{t \in \mathbb{R}}$ is a measurable flow and in the weak closure of the unitary group coming from time $t$ automorphisms we find an operator of the form $f(\mathcal{T})$, where $f: \mathbb{R} \rightarrow \mathbb{C}$ is analytic and different from a multiple of a character, then the flow has the CS property, and hence it has also the JP property. The assumption turns out to be satisfied for many natural examples of flows (see Section 6). In 23, even the simple convolutions property is shown to hold for a large class of smooth flows on the torus.

We show that systems with the JP property are disjoint with ergodically ID systems, which extends the result of [16] for distally simple systems. In particular a consequence is that a typical dynamical system is disjoint from every ergodically ID system. In fact we give a general criterion (Proposition 5) for such a disjointness which is apparently satisfied for a larger class than ergodically ID systems. The criterion applies to systems which can be embedded in symmetric factors of infinitely many Cartesian products, and hence is especially adapted to systems given by 
stationary ID processes (and in particular to Poisson suspension systems). However, we also give simple arguments showing that systems generated by stationary ID processes, such as Poisson suspensions, are factors of ergodically ID systems.

Most results of this paper were obtained during the visit of the first author at the University Paris 13 in Fall 2006.

\section{BASIC DEFINITIONS AND NOTATION}

We will first consider automorphisms acting on standard probability Borel spaces, and their ergodicity will be tacitly assumed. Joinings between automorphisms $T$ and $S$ are meant as measures on the corresponding product space having the coordinate projections as the original measures and invariant under $T \times S$. The set of joinings between these automorphisms is denoted by $J(T, S)$ (with an obvious change of notation when more automorphisms are involved). The subset of ergodic joinings will be denoted by $J^{e}(T, S)$. Following 9 , systems $T$ and $S$, between which the only possible joining is the product measure, are called disjoint; we then write $T \perp S$. If $T$ and $S$ act on $(X, \mathcal{B}, \mu)$ and $(Y, \mathcal{C}, \nu)$ respectively and if $\lambda \in J(T, S)$, then it determines a Markov operator $\Phi_{\lambda}: L^{2}(X, \mathcal{B}, \mu) \rightarrow L^{2}(Y, \mathcal{C}, \nu)$,

$$
\int_{Y} \Phi_{\lambda}(f) g d \nu=\int_{X \times Y} f \otimes g d \lambda, \quad \Phi_{\lambda} \circ T=S \circ \Phi_{\lambda}
$$

(we still denote by $T$ and $S$ the corresponding unitary operators on the $L^{2}$ spaces). $\Phi_{\lambda}$ is doubly stochastic: $\Phi_{\lambda}(1)=\Phi_{\lambda}^{*}(1)=1$, and the image via $\Phi_{\lambda}$ of a non-negative function is non-negative. Notice that $\Phi_{\lambda}(f)=E^{\lambda}(f \otimes 1 \mid Y)$, where by $E^{\lambda}(\cdot \mid Y)$ we denote the conditional expectation with respect to the $\sigma$-algebra $\{\emptyset, X\} \otimes \mathcal{C}$. In fact (1) establishes a 1-1 correspondence between joinings and Markov operators; see e.g. [11], Chapter 6, for more details.

Let $R$ be an ergodic automorphism of a standard probability Borel space $(Z, \mathcal{D}, \rho)$. Following [18, $R$ is said to have the pairwise independence property (PID) if every (finite or infinite) pairwise independent self-joining of $R$ is equal to the product measure. Ryzhikov in [31] proved the following important lemma.

Lemma 1. Assume that $R$ has the PID property and let $T, S$ be automorphisms. Assume that $\lambda \in J(T, S, R)$ is pairwise independent. Then it is the product measure.

For the definition of distality and relative distality we refer the reader to $[10$. An automorphism $R$ is called 2-fold distally simple (see [16]) if each $\lambda \in J^{e}(R, R)$ is either the product measure, or the extension $(R \times R, \lambda)$ is relatively distal over the marginal factors $R$. A 2-fold distal system which has the PID property is called distally simple.

As proved in [16, each 2-fold distally simple automorphism either has purely discrete spectrum or is weakly mixing. Another important property of a 2 -fold distally simple system $R$ is that (see [16]):

If $S$ is an arbitrary automorphism and $\lambda \in J^{e}(R, S)$, then either $\lambda$ is the (2) product measure or the extension $(R \times S, \lambda)$ is relatively distal over the factor

$S$.

For the sake of completeness, since (2) is used in the sequel, we briefly recall its justification from [16]. Denote by $\widehat{\mathcal{C}}$ the maximal factor of $(R \times S, \lambda)$ containing $\{\emptyset, Z\} \otimes \mathcal{C}$ for which $R \times\left. S\right|_{\widehat{\mathcal{C}}} \rightarrow S$ is relatively distal. Then $R \times\left. S\right|_{\mathcal{D} \otimes \mathcal{C}} \rightarrow R \times\left. S\right|_{\widehat{\mathcal{C}}}$ is relatively weakly mixing and the relatively independent square of $(R \times S, \lambda)$ over 
$\widehat{\mathcal{C}}$ is ergodic. We can view this latter system as an ergodic joining $(R \times R \times S, \kappa)$ of two copies of $R$ and $S$. Moreover, by basic properties of relative products (see [10]) the extension $(R \times R \times S, \kappa) \rightarrow(R \times S, \lambda)$ is still relatively weakly mixing.

If $\lambda$ is not the product measure, the two copies of $R$ cannot be independent. Then $\left(R \times R,\left.\kappa\right|_{\mathcal{D} \otimes \mathcal{D}}\right) \rightarrow R_{1}$ is relatively distal and $(R \times R \times S, \kappa) \rightarrow(R \times S, \lambda)$ is also relatively distal. It follows that this extension is trivial and $\widehat{\mathcal{C}}=\mathcal{D} \otimes \mathcal{C}$.

We also recall (see [9]) that

if $T$ is ergodic, the extension $T \rightarrow T_{1}$ is relatively distal and $S$ is weakly

mixing, then the only extension of $T_{1} \times S$ to an ergodic joining of $T$ and $S$

is the direct product.

A dynamical system $T$ will be said to have the convolution singularity (CS) property if its reduced maximal spectral type $\sigma_{T}$ (i.e. the maximal spectral type of $T$ on $L_{0}^{2}(X, \mathcal{B}, \mu)$, the orthocomplement of the constant functions) is singular with respect to the convolution product $\lambda_{1} * \lambda_{2}$ of two arbitrary continuous measures $\lambda_{1}, \lambda_{2}$ on the circle. CS systems have purely singular spectra; thus they automatically have the PID property by the result of Host [12. It can be seen (25]) that the CS property holds if the symmetric tensor product operator $V_{\sigma} \odot V_{\sigma}$ has a simple spectrum, where

$$
V_{\sigma}: L^{2}(\mathbb{T}, \sigma) \rightarrow L^{2}(\mathbb{T}, \sigma), \quad V_{\sigma}(f)(z)=z f(z) .
$$

This relates the CS property of $T$ with spectral properties of the Gaussian automorphism determined by $\sigma_{T}$. In particular, if this Gaussian automorphism has a simple spectrum, then $T$ has the CS property (see [3]).

In what follows, if no confusion arises, we will identify automorphisms with their actions on $\sigma$-algebras; in particular, factor automorphisms will be identified with the underlying invariant sub- $\sigma$-algebras.

\section{Systems WITH JOINING PRIMENESS PROPERTY AND DISJOINTNESS}

An ergodic system $R$ acting on $(Z, \mathcal{D}, \rho)$ is said to have the joining primeness (JP) property if for every pair of weakly mixing systems $S_{1}$ and $S_{2}$ and every $\lambda \in J^{e}\left(R, S_{1} \times S_{2}\right)$ we have

$$
\lambda=\lambda_{Z, Y_{1}} \otimes \nu_{2} \quad \text { or } \quad \lambda=\lambda_{Z, Y_{2}} \otimes \nu_{1},
$$

where by $\lambda_{Z, Y_{i}}$ we denote the projection of $\lambda$ to the corresponding two coordinates.

Of course this definition really makes sense only for a system which is not disjoint from a weakly mixing system.

Notice that assuming $S_{1}$ and $S_{2}$ are isomorphic would give an equivalent definition. Indeed, let $\lambda \in J^{e}\left(R, S_{1} \times S_{2}\right)$, where $S_{1}$ and $S_{2}$ are two weakly mixing systems, not necessarily isomorphic. If $S_{1}^{\prime}$ and $S_{2}^{\prime}$ are isomorphic copies of $S_{1}$ and $S_{2}$ respectively, then, by forming the direct product of the previous joining with the direct product $S_{1}^{\prime} \times S_{2}^{\prime}$, we obtain an ergodic joining of $R$ with the direct product $\left(S_{1} \times S_{2}^{\prime}\right) \times\left(S_{1}^{\prime} \times S_{2}\right)$ of two isomorphic weakly mixing transformations and the equivalence of the definitions follows. Observe also that we could have required $R$ to satisfy a seemingly stronger property: for any $n \geq 2$, any family $S_{1}, \ldots, S_{n}$ of weakly mixing transformations and any $\lambda \in J^{e}\left(R, S_{1} \times \ldots \times S_{n}\right)$, there exists 
$j(\lambda) \in\{1, \ldots, n\}$ such that

$$
\lambda=\lambda_{Z, Y_{j(\lambda)}} \otimes\left(\bigotimes_{j \neq j(\lambda)} \nu_{j}\right) .
$$

Once again, it is not difficult to check that the resulting definition would be equivalent to the previous one and that it is still so if $S_{1}, \ldots, S_{n}$ are assumed isomorphic.

Given $\lambda \in J^{e}\left(R, S_{1} \times \ldots \times S_{n}\right)$, we identify $\mathcal{D}$ and the $\mathcal{C}_{i}$ to sub- $\sigma$-algebras of the joining system. It is easy to see that $\lambda$ satisfies (4) if and only if

$$
\Phi_{\lambda}(f) \in L^{2}\left(\mathcal{C}_{j(\lambda)}\right)
$$

for each $f \in L_{0}^{2}(Z, \mathcal{D}, \rho)$.

Proposition 1. The class of weakly mixing JP systems is closed under factors, inverse limits and distal extensions which are weakly mixing.

Proof. The first two properties are obvious. The fact that the JP property is closed under weakly mixing distal extensions follows from (3).

Let us notice that whenever a JP automorphism $R$ has a common factor with a direct (weakly mixing) product $S_{1} \times S_{2}$, then in fact this common factor is contained in either the first or the second coordinate $\sigma$-algebra of $\mathcal{C}_{1} \otimes \mathcal{C}_{2}$. Hence using del Junco and Rudolph's criterion of non-disjointness of a system from a simple system [18] we obtain the following.

Proposition 2. A JP system is disjoint with a weakly mixing simple system if and only if they do not have a common non-trivial factor.

The result below is proved in [33] and [16] in a slightly different form, and we give a proof only for completeness.

Proposition 3. If $R$ is weakly mixing and distally simple, then it has the JP property.

Proof. Assume that $\lambda \in J^{e}\left(R, S_{1} \times S_{2}\right)$. By (2), the extension $\left(R \times S_{1}, \lambda_{Z, Y_{1}}\right) \rightarrow S_{1}$ is either relatively distal or we have independence. If it is relatively distal, since $S_{2}$ is weak mixing, we have $\lambda=\lambda_{Z, Y_{1}} \otimes \nu_{2}$ by (3). Similarly, if $\left(R \times S_{2}, \lambda_{Z, Y_{2}}\right) \rightarrow S_{1}$ is relatively distal, we get $\lambda=\lambda_{Z, Y_{2}} \otimes \nu_{1}$. Furthermore, if $\mathcal{D}$ is independent from $\mathcal{C}_{1}$ and $\mathcal{C}_{2}$, then by the PID property of $R$ (and Lemma 1), $\lambda$ is just the product measure.

In order to prove that CS implies JP we need a lemma.

Lemma 2. Assume that $\lambda \in J^{e}\left(R, S_{1} \times S_{2}\right)$ and that it satisfies

$$
\Phi_{\lambda}\left(L_{0}^{2}(Z, \mathcal{D}, \rho)\right) \subset L_{0}^{2}\left(Y_{1}, \mathcal{C}_{1}, \nu_{1}\right) \oplus L_{0}^{2}\left(Y_{2}, \mathcal{C}_{2}, \nu_{2}\right) .
$$

Then $\lambda=\lambda_{Z, Y_{1}} \otimes \nu_{2}$ or $\lambda=\lambda_{Z, Y_{2}} \otimes \nu_{1}$.

Proof. Denoting by $p_{i}$ the orthogonal projection from $L^{2}\left(Y_{1} \times Y_{2}, \nu_{1} \otimes \nu_{2}\right)$ on $L^{2}\left(Y_{i}, \nu_{i}\right)$ and by $E$ the orthogonal projection on constant functions, by the assumption we have

$$
\Phi_{\lambda}+E=p_{1} \circ \Phi_{\lambda}+p_{2} \circ \Phi_{\lambda} .
$$


But $p_{i} \circ \Phi_{\lambda}$ is still a Markov operator, so $p_{i} \circ \Phi_{\lambda}=\Phi_{\lambda_{i}}$ for some joining $\lambda_{i}, i=1,2$. Now the condition in (6) simply means that

$$
\lambda+\mu \otimes \nu_{1} \otimes \nu_{2}=\lambda_{Z, Y_{1}} \otimes \nu_{2}+\lambda_{Z, Y_{2}} \otimes \nu_{1},
$$

and since the four joinings under consideration are ergodic (the factor joinings $\lambda_{Z, Y_{i}}$ are ergodic and the $S_{i}$ are weakly mixing), the assertion follows.

Proposition 4. If $R$ is weakly mixing and has the CS property, then it has the JP property.

Proof. For each $f \in L^{2}(Z, \mathcal{D}, \rho)$, it is a general fact that the spectral measure of $\Phi_{\lambda}(f)$ is absolutely continuous with respect to the spectral measure of $f$ and therefore with respect to $\sigma_{R}$. Since

$$
L_{0}^{2}\left(Y_{1} \times Y_{2}, \nu_{1} \otimes \nu_{2}\right)=L_{0}^{2}\left(Y_{1}, \nu_{1}\right) \oplus L_{0}^{2}\left(Y_{2}, \nu_{2}\right) \oplus\left(L_{0}^{2}\left(Y_{1}, \nu_{1}\right) \otimes L_{0}^{2}\left(Y_{2}, \nu_{2}\right)\right)
$$

and on $L_{0}^{2}\left(Y_{1}, \nu_{1}\right) \otimes L_{0}^{2}\left(Y_{2}, \nu_{2}\right)$ the maximal spectral type is the convolution of the reduced maximal spectral types of $S_{1}$ and $S_{2}$, we obtain that the assumption of Lemma 2 is satisfied. Therefore, the result follows.

We will give examples of automorphisms with the CS property in Section 6

\section{JOINING PRIMENESS PROPERTY AND SYMMETRIC FACTORS}

The aim of this section is to prove that JP systems are disjoint from dynamical systems that possess the probabilistic property of infinite divisibility (not to be mistaken with the ergodic theoretical notion sharing the same name; see the next section).

Let us recall the definition of an infinitely divisible (ID) stationary process:

A stationary process $\left\{X_{n}\right\}_{n \in \mathbf{Z}}$ of distribution $P$ on $\left(\mathbf{R}^{\mathbf{Z}}, \mathcal{B}^{\otimes \mathbf{Z}}\right)$ is ID if, for any integer $k$, there exists a probability distribution $P_{k}$ on $\left(\mathbf{R}^{\mathbf{Z}}, \mathcal{B}^{\otimes \mathbf{Z}}\right)$ such that $P=$ $P_{k} * \cdots * P_{k}$ ( $k$ terms $)$, where $*$ denotes the convolution operation with respect to the usual coordinatewise addition on $\mathbf{R}^{\mathbf{Z}}$. In other words, the stationary process $\left\{X_{n}\right\}_{n \in \mathbf{Z}}$ is ID if, for any integer $k$, it can be realized as an independent sum $X_{n}=X_{n}^{(1, k)}+\cdots+X_{n}^{(k, k)}$, where the processes $\left\{X_{n}^{(i, k)}\right\}_{n \in \mathbb{Z}}$ are stationary with the same distribution $P_{k}$. If $P$ is infinitely divisible, then the distributions $P_{k}$ are uniquely determined and are also infinitely divisible. Moreover if $\left(\mathbf{R}^{\mathbf{Z}}, \mathcal{B}^{\otimes \mathbf{Z}}, P, S\right)$ (where $S$ stands for the shift) is ergodic, then it is weakly mixing (see 28]) and so is $\left(\mathbf{R}^{\mathbf{Z}}, \mathcal{B}^{\otimes \mathbf{Z}}, P_{k}, S\right)$ for any $k$. Any Gaussian stationary process is infinitely divisible as well as any stable stationary process among many others.

The important ergodic property here that will play the key role for our disjointness result comes from the following observation: the system determined by a stationary ID process is, for any integer $k \geq 1$, a factor of the symmetric factor of the Cartesian $k$-th power of a dynamical system (by symmetric factor we mean the sub- $\sigma$-algebra of subsets which are invariant under all permutations of coordinates). Indeed, $\left(\mathbf{R}^{\mathbf{Z}}, \mathcal{B}^{\otimes \mathbf{Z}}, P, S\right)$ is a factor of $\left(\mathbf{R}^{\mathbf{Z}}, \mathcal{B}^{\otimes \mathbf{Z}}, P_{k}, S\right)^{\times k}$ via the factor $\operatorname{map}\left(\left\{x_{n}^{1}\right\}_{n \in \mathbf{Z}}, \ldots,\left\{x_{n}^{k}\right\}_{n \in \mathbf{Z}}\right) \mapsto\left\{x_{n}^{1}+\cdots+x_{n}^{k}\right\}_{n \in \mathbf{Z}}$.

Closely related to ID stationary processes are Poisson suspensions. Let us recall the definition.

Consider a dynamical system $(X, \mathcal{A}, \mu, T)$, where $\mu$ is $\sigma$-finite and form the Poisson measure $\left(X^{*}, \mathcal{A}^{*}, \mu^{*}\right)$, where $X^{*}$ is the space of counting measures on $(X, \mathcal{A})$, 
$\mathcal{A}^{*}$ the smallest $\sigma$-algebra such that for any $A \in \mathcal{A}^{*}$ the map $N_{A}: \nu \mapsto \nu(A)$ is measurable and $\mu^{*}$ is the unique probability measure such that for any family $A_{1}, \ldots, A_{k}$ of disjoint sets in $\mathcal{A}$ of finite $\mu$-measure, the random variables $N_{A_{i}}$ are independent and Poisson distributed with parameters $\mu\left(A_{i}\right)$. Furthermore let $T_{*}: X^{*} \rightarrow X^{*}$ be the map which assigns to each $\nu \in X^{*}$ its direct image under $T$. Then $T_{*}$ is $\mu^{*}$-preserving and $\left(X^{*}, \mathcal{A}^{*}, \mu^{*}, T_{*}\right)$ is called the Poisson suspension over the base $(X, \mathcal{A}, \mu, T)$ (see [3], and also the recent publications [5], 29], 30] for the joining theory of Poisson suspensions). Convolution in this space is well defined, arising from the addition of measures. It is well known that $\mu^{*}=\left(\frac{1}{k} \mu\right)^{*} * \cdots *\left(\frac{1}{k} \mu\right)^{*}$ ( $k$ terms) for every $k \geq 1$, and this shows the ID character of a Poisson suspension. We therefore derive the same conclusion as above: for any integer $k$ a Poisson suspension is a factor of the symmetric factor of a direct product of $k$ copies of a dynamical system.

The disjointness of JP systems from systems given by ID stationary processes and Poisson suspensions is based on the relationship between JP systems and symmetric factors. In fact, it will be a consequence of a bit more general criterion given in Proposition 5 below. We first need a lemma.

Let $S$ be a weakly mixing automorphism of a standard probability space $(Y, \mathcal{C}, \nu)$. Given $n \geq 1$, by $\mathcal{F}_{n}=\mathcal{F}_{n}(\mathcal{C})=\mathcal{F}_{n}(S)$ we denote the symmetric factor of $S^{\times n}$.

Proposition 5. Let $T$ be an automorphism of a standard probability space $(X, \mathcal{B}, \mu)$. Assume that, for each $g \in L^{2}(X, \mathcal{B}, \mu)$, there exist a sequence $\left(k_{j}\right)_{j \geq 1}$ of integers going to infinity and a sequence $\left(S_{j}\right)_{j \geq 1}$ of weakly mixing automorphisms such that $T$ is a factor of $S_{j}^{\times k_{j}}$ and moreover

$$
\operatorname{dist}\left(g, L^{2}\left(\mathcal{F}_{k_{j}}\left(S_{j}\right)\right)=o\left(\frac{1}{\sqrt{k_{j}}}\right)\right.
$$

Then $T$ is disjoint from every weakly mixing JP system.

Proof. Let $\lambda_{0} \in J(R, T)$, where $R:(Z, \mathcal{D}, \rho) \rightarrow(Z, \mathcal{D}, \rho)$ is a JP automorphism and let $f$ be any function in $L_{0}^{2}(Z, \mathcal{D}, \rho)$. We have to prove that $g:=\Phi_{\lambda_{0}}(f)=0$.

By the assumption, given an arbitrary $\varepsilon>0$, there exist an arbitrarily large integer $k>0$ and a weakly mixing automorphism $S$ on a standard space $(Y, \mathcal{C}, \nu)$ such that $T$ is a factor of $S^{\times k}$ and moreover

$$
\operatorname{dist}\left(g, L^{2}\left(\mathcal{F}_{k}(S)\right) \leq \frac{\varepsilon}{\sqrt{k}} .\right.
$$

Let $\widetilde{\lambda}_{0}$ be the relatively independent extension of $\lambda_{0}$ to a joining of $R$ with $S^{\times k}$, so that $\Phi_{\widetilde{\lambda}_{0}}$ is the composition of $\Phi_{\lambda_{0}}$ and the embedding of $L^{2}(X, \mathcal{B}, \mu)$ into $L^{2}\left(Y^{\times n}, \mathcal{C}^{\otimes n}, \nu^{\otimes n}\right)$, and thus $\Phi_{\widetilde{\lambda}_{0}}(f)=\Phi_{\lambda_{0}}(f)=g$.

Consider now an ergodic decomposition of $\widetilde{\lambda}_{0}$ :

$$
\widetilde{\lambda}_{0}=\int_{J^{e}\left(R, S^{\times k}\right)} \lambda d P(\lambda) .
$$

For $\lambda \in J^{e}\left(R, S^{\times k}\right)$, we write the underlying $\sigma$-algebra as $\mathcal{D} \vee \mathcal{C}_{1} \vee \ldots \vee \mathcal{C}_{k}$, where the $\mathcal{C}_{i}$ 's algebras remain independent. When $\Phi_{\lambda}(f) \neq 0$, since $R$ has the JP property, there exists exactly one $1 \leq j=j(\lambda) \leq k$ such that $\Phi_{\lambda}(f) \in L^{2}\left(\mathcal{C}_{j(\lambda)}\right)$. 
For $1 \leq j \leq k$, denote by $M_{j}$ the set of those $\lambda \in J^{e}\left(R, S^{\times k}\right)$ for which $\Phi_{\lambda}(f) \neq 0$ and $j(\lambda)=j$. The sets $M_{j}$ are disjoint and clearly measurable. Let

$$
f_{j}:=\int_{M_{j}} \Phi_{\lambda}(f) d P \in L^{2}\left(\mathcal{C}_{j}\right) .
$$

We obtain the orthogonal decomposition

$$
g=\int_{J^{e}\left(R, S^{\times k}\right)} \Phi_{\lambda}(f) d P=\sum_{j=1}^{k} f_{j} \in \bigoplus_{j=1}^{k} L^{2}\left(\mathcal{C}_{j}\right),
$$

and moreover there exists some $j_{0} \in\{1, \ldots, k\}$ such that $P\left(M_{j_{0}}\right) \leq \frac{1}{k}$, whence

$$
\left\|f_{j_{0}}\right\| \leq \frac{1}{k}\|f\| \text {. }
$$

Now we claim that for all $1 \leq j \leq k$,

$$
\left|\left\|f_{j}\right\|-\left\|f_{j_{0}}\right\|\right| \leq \frac{2 \varepsilon}{\sqrt{k}} .
$$

Indeed, consider a permutation $\pi$ of $\{1, \ldots, k\}$ sending $j_{0}$ to $j$ and the corresponding unitary operator $U_{\pi}$ on $L^{2}\left(Y^{\times n}, \mathcal{C}^{\otimes n}, \nu^{\otimes n}\right)$. Since all functions in $L^{2}\left(\mathcal{F}_{k}(S)\right)$ are fixed by $U_{\pi}$,

$$
\left\|f_{j}-f_{j_{0}}\right\| \leq\left(\sum_{\ell=1}^{k}\left\|f_{\pi(\ell)}-f_{\ell}\right\|_{L^{2}(\mathcal{C})}^{2}\right)^{1 / 2}=\left\|U_{\pi}(g)-g\right\| \leq 2 \operatorname{dist}\left(g, L^{2}\left(\mathcal{F}_{k}(S)\right)\right.
$$

and (9) follows from (7).

So, by (8), $\left\|f_{j}\right\| \leq \frac{1}{k}\|f\|+\frac{2 \varepsilon}{\sqrt{k}}$ for all $1 \leq j \leq k$ and

$$
\|g\|=\left(\sum_{j=1}^{k}\left\|f_{j}\right\|^{2}\right)^{1 / 2} \leq \frac{1}{\sqrt{k}}\|f\|+2 \varepsilon
$$

Since $k$ was arbitrarily large and $\varepsilon$ was arbitrarily small, this proves that $g=0$.

Remark 1. We notice that T satisfying the assumption of Proposition 5 is still disjoint from all roots of JP maps. Indeed, if $T$ satisfies the assumptions of Proposition 5, then each (non-zero) power of it does so; moreover if two automorphisms are non-disjoint, then also their powers are non-disjoint and the disjointness result with roots of JP maps follows.

\section{JP PROPERTY AND INFINITE DIVISIBILITY IN THE ERGODIC THEORETICAL SENSE}

Let us recall that an ergodic automorphism $T$ is said to be infinitely divisible if there exists a sequence of factors $\left\{\mathcal{B}_{\omega}: \omega \in\{0,1\}^{*}\right\}$ of $\mathcal{B}$ where $\mathcal{B}_{\varepsilon}=\mathcal{B}$, $\mathcal{B}_{\omega}=\mathcal{B}_{\omega 0} \otimes \mathcal{B}_{\omega 1}$ and for each $f \in L_{0}^{2}(X, \mathcal{B}, \mu), \eta \in\{0,1\}^{\mathbb{N}}$,

$$
\lim _{n \rightarrow \infty} E\left(f \mid \mathcal{B}_{\eta[0, n)}\right)=0 \text {. }
$$

As in the introduction, to avoid confusion, this ergodic theoretical notion of infinite divisibility will be referred to as ergodically $I D$ in the sequel.

Proposition 6. Ergodically ID automorphisms are disjoint from JP systems. 
Proof. Assume that $R$ is a JP automorphism, and let $\lambda \in J^{e}(R, T)$, where $T$ is ergodically ID. For each $f \in L_{0}^{2}(Z, \mathcal{D}, \rho)$ and $n \geq 1$, by the JP property of $R$, there is an $\omega \in\{0,1\}^{n}$ such that $\Phi_{\lambda}(f)$ belongs to $L^{2}\left(\mathcal{B}_{\omega}\right)$ (indeed, $\mathcal{B}=\bigotimes_{|\omega|=n} \mathcal{B}_{\omega}$ ). Then the result follows immediately from (10).

In order to complete the picture we will now give a general argument that in particular shows that dynamical systems induced by stationary ID processes are factors of ergodically ID dynamical systems.

Proposition 7. Let $T$ be an automorphism of a standard probability space $(X, \mathcal{B}, \mu)$. Assume that there exists a sequence $\left(S_{n}\right)_{n \geq 0}$ of automorphisms of standard probability spaces $\left(Y_{n}, \mathcal{C}_{n}, \nu_{n}\right)$ such that $S_{0}=T$ and, for every $n \geq 0, S_{n}$ is isomorphic to a factor of the symmetric factor of $S_{n+1} \times S_{n+1}$. Then $T$ is a factor of an ergodically ID system.

Proof. Let, at each stage $n \geq 0, T_{n}:=\Pi_{\omega \in\{0,1\}^{n}} S_{\omega}$ be the direct product of $2^{n}$ copies $S_{\omega}$ of $S_{n}$ each acting on a space $\left(Y_{\omega}, \mathcal{C}_{\omega}, \nu_{\omega}\right)$, and $T_{n}$ acting on the product space $\left(X_{n}, \mathcal{B}_{n}, \mu_{n}\right)$. From the assumption we represent each $S_{\omega}$ as a factor of the symmetric factor of $S_{\omega 0} \times S_{\omega 1}$. So each $T_{n}$ is a factor of $T_{n+1}$, and we obtain an inverse system of dynamical systems. Let $\widetilde{T}$ stand for the inverse limit, acting on $(\widetilde{X}, \widetilde{\mathcal{B}}, \widetilde{\mu})$. We will show that $\widetilde{T}$ is ergodically ID.

We consider each $\mathcal{B}_{n}$ and $\mathcal{C}_{\omega}$ as sub- $\sigma$-algebras of $\widetilde{\mathcal{B}}$ : then $\widetilde{\mathcal{B}}$ is generated by the union of the increasing sequence of factors $\mathcal{B}_{n}$, and each $\mathcal{C}_{\omega}$ is contained in the symmetric factor $\mathcal{F}_{2}\left(\mathcal{B}_{\omega 0}, \mathcal{B}_{\omega 1}\right)$. Moreover, for $\omega \in\{0,1\}^{*}$, let

$$
\mathcal{B}_{\omega}=\bigvee_{\eta \in\{0,1\}^{*}} \mathcal{C}_{\omega \eta}
$$

We obtain a splitting sequence of independent factors as required in the definition of an ergodically ID system. We need to show that (10) holds true, and it is enough here to consider $f \in L_{0}^{2}\left(\mathcal{B}_{n}\right)$ for some $n \geq 0$. Then, for $\omega \in\{0,1\}^{n}$,

$$
E\left(f \mid \mathcal{B}_{\omega}\right)=E\left(f \mid \mathcal{C}_{\omega}\right) .
$$

Indeed, it is again enough here to consider $f$ of the form $f=\bigotimes_{\omega^{\prime} \in\{0,1\}^{n}} f_{\omega^{\prime}}$ with $f_{\omega}^{\prime}$ measurable with respect to $\mathcal{C}_{\omega^{\prime}}$, and then $f_{\omega^{\prime}}$ is still independent from $\mathcal{B}_{\omega}$ whenever $\omega^{\prime} \neq \omega$.

Now, represent $E\left(f \mid \mathcal{C}_{\omega}\right)$ as an orthogonal sum

$$
E\left(f \mid \mathcal{C}_{\omega}\right)=E\left(f \mid \mathcal{C}_{\omega 0}\right)+E\left(f \mid \mathcal{C}_{\omega 1}\right)+g .
$$

Since $\mathcal{C}_{\omega}$ is contained in the symmetric factor of $S_{\omega 0} \times S_{\omega 1}$, we have that $E\left(f \mid \mathcal{C}_{\omega 1}\right)$ corresponds to $E\left(f \mid \mathcal{C}_{\omega 0}\right)$ by the given isomorphism between $S_{\omega 0}$ and $S_{\omega 1}$, and in particular it has the same norm. Therefore

$$
\left\|E\left(f \mid \mathcal{C}_{\omega i}\right)\right\| \leq \frac{1}{\sqrt{2}}\left\|E\left(f \mid \mathcal{C}_{\omega}\right)\right\| \leq \frac{1}{\sqrt{2}}\|f\|
$$

for $i=0,1$.

We may repeat the same argument, and thus we obtain for every $\eta \in\{0,1\}^{*}$ :

$$
\left\|E\left(f \mid \mathcal{C}_{\omega \eta}\right)\right\| \leq\left(\frac{1}{\sqrt{2}}\right)^{|\eta|}\|f\| .
$$

Moreover, since $\mathcal{B}_{n} \subset \mathcal{B}_{p}$ for $p \geq n$, we still have $E\left(f \mid \mathcal{B}_{\omega \eta}\right)=E\left(f \mid \mathcal{C}_{\omega \eta}\right)$ by (11) and the result follows. 
Applying this result to a Poisson suspension $\left(X^{*}, \mathcal{A}^{*}, \mu^{*}, T_{*}\right)$ and following the lines of the construction, it can be checked that the inverse limit is indeed isomorphic to the Poisson suspension of

$$
(X \times[0,1), \mathcal{A} \otimes \mathcal{B}([0,1)), \mu \otimes \lambda, T \times I d),
$$

where $\lambda$ is Lebesgue measure on $[0,1)$. Indeed, we recall that $\left(X^{*}, \mathcal{A}^{*}, \mu^{*}, T_{*}\right)$ is a factor of the symmetric factor of $\left(X^{*}, \mathcal{A}^{*},\left(\frac{1}{2} \mu\right)^{*}, T_{*}\right)^{\times 2}$, but this latter system is isomorphic to the Poisson suspension of

$$
\left(X \times\{0,1\}, \mathcal{A} \otimes \mathcal{P}(\{0,1\}), \mu \otimes\left(\frac{1}{2} \delta_{0}+\frac{1}{2} \delta_{1}\right), T \times I d\right),
$$

and the result follows, proceeding inductively.

\section{REMARKS CONCERNING JP PROPERTY OF HIGHER ORDER}

We can extend the definition of JP, considering a weaker property for multiple joinings: an ergodic system $R$ is said to have the joining primeness property of order $n \geq 1(\mathrm{JP}(n))$ if for every $k \geq n+1$, every direct product of $k$ weakly mixing automorphisms $S_{1}, \ldots, S_{k}$, and every $\lambda \in J^{e}\left(R, S_{1} \times \ldots \times S_{k}\right)$, there exist $i_{1}, \ldots, i_{n}$ in $\{1, \ldots, k\}$ such that

$$
\lambda=\lambda_{Z, Y_{i_{1}}, \ldots, Y_{i_{n}}} \otimes \bigotimes_{j \neq i_{1}, \ldots, i_{n}} \nu_{j}
$$

Clearly $\mathrm{JP}(m) \subseteq \mathrm{JP}(n)$ whenever $m \leq n$ and $\mathrm{JP}(1)$ is just the class JP considered in this paper. Note that, as in this latter case, we can replace in the definition possibly non-isomorphic $S_{i}$ 's by isomorphic ones.

Proposition 8. JP(n) systems are disjoint from ergodically ID systems.

Proof. Let $R$ belong to the $\operatorname{JP}(n)$ class, $T$ be ergodically ID and consider an ergodic joining $\lambda$ of $R$ and $T$ and the corresponding Markov operator $\Phi_{\lambda}: L^{2}(Z, \mathcal{D}, \rho) \rightarrow$ $L^{2}(X, \mathcal{B}, \mu)$.

With the notation of the previous section, let $\Omega_{k}$ be the set of all $\omega \in\{0,1\}^{*}$ with $|\omega|=k$, for $k \geq 0$, so that $\mathcal{B}=\bigotimes_{\omega \in \Omega_{k}} \mathcal{B}_{\omega}$. By the $\operatorname{JP}(n)$ property of $R$, we can find subsets $\Delta$ of $\Omega_{k}$ of cardinality $\leq n$ such that $\lambda$ is the product of its restriction to $\mathcal{D} \vee \bigotimes_{\omega \in \Delta} \mathcal{B}_{\omega}$ and the other factors or, equivalently,

$$
\Phi_{\lambda}\left(L^{2}(\mathcal{D})\right) \subset L^{2}\left(\bigotimes_{\omega \in \Delta} \mathcal{B}_{\omega}\right)
$$

Since, for $\Delta, \Delta^{\prime} \subset \Omega_{k}$, we have by independence

$$
L^{2}\left(\bigotimes_{\omega \in \Delta} \mathcal{B}_{\omega}\right) \cap L^{2}\left(\bigotimes_{\omega \in \Delta^{\prime}} \mathcal{B}_{\omega}\right)=L^{2}\left(\bigotimes_{\omega \in \Delta \cap \Delta^{\prime}} \mathcal{B}_{\omega}\right),
$$

it follows that there is a smallest set $\Delta \subset \Omega_{k}$ satisfying (12), which we denote by $\Omega_{k}^{\lambda}$. Also let $n_{k}$ be its cardinality $\left(n_{k} \leq n\right.$ for all $\left.k\right)$.

Now, from $\mathcal{B}_{\omega}=\mathcal{B}_{\omega 0} \otimes \mathcal{B}_{\omega 1}$ for all $\omega$, it follows that $\Omega_{k}^{\lambda}$ is contained in the set of all $\omega[0, k)$ for $\omega \in \Omega_{k+1}^{\lambda}$, and conversely $\Omega_{k+1}^{\lambda}$ is contained in the set of all $\omega 0$ and all $\omega 1$ for $\omega \in \Omega_{k}^{\lambda}$. In particular, $n_{k}$ is non-decreasing, and, being bounded by $n$, it is eventually constant. This implies that there exists $K$ such that for $k \geq K$, 
$n_{k}=n_{K}$ and for each $\omega \in \Omega_{k}^{\lambda}$ there is exactly one $\omega^{\prime} \in\{\omega 0, \omega 1\}$ in $\Omega_{k+1}^{\lambda}$. Hence there exist $\eta_{1}, \ldots, \eta_{n_{K}}$ in $\{0,1\}^{\mathbb{N}}$ such that

$$
\Phi_{\lambda}\left(L^{2}(\mathcal{D})\right) \subset \bigcap_{k \geq K} L^{2}\left(\bigotimes_{i=1}^{n_{K}} \mathcal{B}_{\eta_{i}[0, k)}\right) .
$$

Finally, again by independence, for each $k \geq K$, the orthogonal projection from $L^{2}\left(\bigotimes_{i=1}^{n_{K}} \mathcal{B}_{\eta_{i}[0, K)}\right)$ onto $L^{2}\left(\bigotimes_{i=1}^{n_{K}} \mathcal{B}_{\eta_{i}[0, k)}\right)$ is the tensor product of the orthogonal projections of the factors. By (10) for each $i \in\left\{1, \ldots, n_{K}\right\}$ the projection of every $f \in L_{0}^{2}\left(\mathcal{B}_{\eta_{i}[0, K)}\right)$ in $L^{2}\left(\mathcal{B}_{\eta_{i}[0, k)}\right)$ goes to zero as $k$ tends to infinity, and it follows that the same holds for the tensor product. Therefore $\Phi_{\lambda}$ is the orthogonal projection onto the constant functions and $\lambda$ is the product measure.

5.1. Examples. We present here a family of systems which are $\operatorname{JP}(2)$ but not $\mathrm{JP}(1)$. Recall that a direct product of two weakly mixing automorphisms cannot have the JP property, so we get such examples from the proposition below if we assume moreover the automorphisms to be weakly mixing.

Proposition 9. If $R_{1}$ has the JP property and $R_{2}$ is distally simple, then $R_{1} \times R_{2}$ is $J P(2)$.

Proof. Let $S_{1}, S_{2}$ and $S_{3}$ be weakly mixing automorphisms and consider an ergodic joining $\lambda$ between $R_{1} \times R_{2}$ and $S_{1} \times S_{2} \times S_{3}$. In this joining we denote by $\mathcal{D}_{i}$ and $\mathcal{C}_{j}$ the factor $\sigma$-algebra corresponding to each transformation $R_{i}$ and $S_{j}$.

By the JP property of $R_{1}$ we can assume, up to exchange of coordinates, that $\mathcal{D}_{1} \vee \mathcal{C}_{1}$ is independent from $\mathcal{C}_{2} \vee \mathcal{C}_{3}$, i.e. that $\mathcal{D}_{1} \vee \mathcal{C}_{1}, \mathcal{C}_{2}$ and $\mathcal{C}_{3}$ are independent. Since $R_{2}$ also has the JP property, $\mathcal{D}_{2}$ is independent from either $\mathcal{C}_{2}$ or $\mathcal{C}_{3}$ and we may assume $\mathcal{D}_{2} \perp \mathcal{C}_{3}$.

Now, $\mathcal{D}_{1} \vee \mathcal{C}_{1} \vee \mathcal{C}_{2}$ is independent from $\mathcal{C}_{3}$ and, since $R_{2}$ is distally simple, by (2), either $\mathcal{D}_{1} \vee \mathcal{D}_{2} \vee \mathcal{C}_{1} \vee \mathcal{C}_{2}$ is relatively distal over $\mathcal{D}_{1} \vee \mathcal{C}_{1} \vee \mathcal{C}_{2}$, or $\mathcal{D}_{2}$ is independent from $\mathcal{D}_{1} \vee \mathcal{C}_{1} \vee \mathcal{C}_{2}$. In the first case, since $S_{3}$ is weakly mixing, we get by (3) that $\mathcal{D}_{1} \vee \mathcal{D}_{2} \vee \mathcal{C}_{1} \vee \mathcal{C}_{2}$ remains independent from $\mathcal{C}_{3}$. In the latter case, $\mathcal{D}_{1} \vee \mathcal{C}_{1} \vee \mathcal{C}_{2}$, $\mathcal{D}_{2}$ and $\mathcal{C}_{3}$ are pairwise independent, whence independent by the PID property of $R_{2}$ and Lemma 1, and we get again $\mathcal{D}_{1} \vee \mathcal{D}_{2} \vee \mathcal{C}_{1} \vee \mathcal{C}_{2} \perp \mathcal{C}_{3}$.

\section{Flows With The CONVOLUTION SINGULARITY PROPERTY}

As noted in the introduction, all definitions and results of this paper extend to the case of flows. In this section we will deal with flows since special flows over irrational rotations can often be shown to satisfy the assumptions of Proposition 10 below. Besides, it is not hard to see that a flow $\left(T_{t}\right)_{t \in \mathbb{R}}$ has the CS property if and only if any given non-zero time $t$ automorphism of it has the CS property.

Assume that $\left(T_{t}\right)_{t \in \mathbb{R}}$ is a (measurable) measure-preserving flow of $(X, \mathcal{B}, \mu)$ and let $\sigma$ be its reduced maximal spectral type. We also denote by $\left(T_{t}\right)$ the associated unitary one-parameter group on $L^{2}(X, \mathcal{B}, \mu)$. Then, given $t \in \mathbb{R}, T_{t}$ on $L_{0}^{2}$ corresponds spectrally to the multiplication by $s \mapsto e^{2 \pi i t s}$ on $L^{2}(\mathbb{R}, \sigma)$, and if we define the operator $V$ on $L^{2}(X, \mathcal{B}, \mu)$ as the weak limit of a sequence $\left(T_{t_{n}}\right)_{n \in \mathbb{N}}, V$ corresponds to the multiplication by a function $\phi_{V}$ (being the weak-* limit of the sequence $\left(e^{2 \pi i t_{n} \cdot}\right)$ in $\left.L^{\infty}(\mathbb{R}, \sigma)\right)$. 
Proposition 10. Assume that there exists a sequence $\left(T_{t_{n}}\right)_{n \in \mathbb{N}}$ converging weakly to $V$ which is not of the form $c T_{s}$ and such that $\phi_{V}$ is analytic. Then $\sigma$ is singular with respect to the convolution of any two continuous measures.

Proof. Let us notice first that the result is purely spectral: If $\sigma \not \perp \lambda_{1} * \lambda_{2}$, where $\lambda_{i}$ are positive continuous Borel measures on $\mathbb{R}$, and if $e^{2 \pi i t_{n} \cdot} \rightarrow \phi$ weakly-* in $L^{\infty}(\mathbb{R}, \sigma)$, where $\phi$ is analytic, then $\phi$ must be a multiple of a character.

Since $e^{2 \pi i t_{n} \cdot} \rightarrow \phi_{V}$ in $L^{\infty}(\mathbb{R}, \sigma)$ implies the same weak-* convergence in $L^{\infty}(\mathbb{R}, \eta)$ for each $\eta \ll \sigma$, w.l.o.g. we can assume that $0<\sigma \ll \lambda_{1} * \lambda_{2}$ and moreover that $\phi_{V}$ is not identically zero. By passing to a subsequence of $\left(t_{n}\right)$ if necessary and thanks to the weak-* compactness, $e^{2 \pi i t_{n}}$. converges weakly-* to $\psi_{1}, \psi_{2}$ and $\psi$ in $L^{\infty}\left(\mathbb{R}, \lambda_{1}\right), L^{\infty}\left(\mathbb{R}, \lambda_{2}\right)$ and $L^{\infty}\left(\mathbb{R}, \lambda_{1} * \lambda_{2}\right)$ respectively, where $\psi_{i}$ and $\psi$ are Borel functions which we assume to be defined everywhere. We thus obtain the "generalized character" property

$$
\psi\left(s_{1}+s_{2}\right)=\psi_{1}\left(s_{1}\right) \psi_{2}\left(s_{2}\right)
$$

for $\lambda_{1} \otimes \lambda_{2}$-almost all $\left(s_{1}, s_{2}\right) \in \mathbb{R} \times \mathbb{R}$. This can be found in [13, but we give a proof for completeness. For $f_{i} \in L^{\infty}\left(\mathbb{R}, \lambda_{i}\right)$ we put $d \lambda_{i}^{\prime}=f_{i} d \lambda_{i}$; we have

$$
\begin{gathered}
\int_{\mathbb{R}} e^{2 \pi i t_{n} s} d \lambda_{1}^{\prime} * \lambda_{2}^{\prime}(s)=\int_{\mathbb{R} \times \mathbb{R}} e^{2 \pi i t_{n}\left(s_{1}+s_{2}\right)} f_{1}\left(s_{1}\right) f_{2}\left(s_{2}\right) d \lambda_{1}\left(s_{1}\right) d \lambda_{2}\left(s_{2}\right) \\
=\int_{\mathbb{R}} e^{2 \pi i t_{n} s_{1}} f_{1}\left(s_{1}\right) d \lambda_{1}\left(s_{1}\right) \int_{\mathbb{R}} e^{2 \pi i t_{n} s_{2}} f_{2}\left(s_{2}\right) d \lambda_{2}\left(s_{2}\right) .
\end{gathered}
$$

Thus, by passing to the weak-* limits,

$$
\int_{\mathbb{R}} \psi(s) d \lambda_{1}^{\prime} * \lambda_{2}^{\prime}(s)=\int_{\mathbb{R}} \psi_{1}\left(s_{1}\right) d \lambda_{1}^{\prime}\left(s_{1}\right) \int_{\mathbb{R}} \psi_{2}\left(s_{2}\right) d \lambda_{2}^{\prime}\left(s_{2}\right) .
$$

It follows that

$$
\int_{\mathbb{R} \times \mathbb{R}} \psi\left(s_{1}+s_{2}\right) d \lambda_{1}^{\prime}\left(s_{1}\right) d \lambda_{2}^{\prime}\left(s_{2}\right)=\int_{\mathbb{R}} \psi_{1}\left(s_{1}\right) d \lambda_{1}^{\prime}\left(s_{1}\right) \int_{\mathbb{R}} \psi_{2}\left(s_{2}\right) d \lambda_{2}^{\prime}\left(s_{2}\right),
$$

whence (13) holds since functions of the form $f_{1} \otimes f_{2}$ generate a linearly dense subset of $L^{1}\left(\mathbb{R} \times \mathbb{R}, \lambda_{1} \otimes \lambda_{2}\right)$.

Of course, we also have $\psi=\phi_{V} \sigma$-almost everywhere. Put $h=\frac{d \sigma}{d \lambda_{1} * \lambda_{2}}$ and define the measure $\tilde{\sigma}$ on $\mathbb{R} \times \mathbb{R}$ by $d \tilde{\sigma}=\tilde{h} d \lambda_{1} \otimes \lambda_{2}$, where $\tilde{h}\left(s_{1}, s_{2}\right)=h\left(s_{1}+s_{2}\right)$. Then by (13) and the definition of $\tilde{\sigma}$,

$$
\phi_{V}\left(s_{1}+s_{2}\right)=\psi_{1}\left(s_{1}\right) \psi_{2}\left(s_{2}\right)
$$

for $\tilde{\sigma}$-almost all $\left(s_{1}, s_{2}\right) \in \mathbb{R}^{2}$.

Put $C=\left\{\left(s_{1}, s_{2}\right) \in \mathbb{R}^{2}: \phi_{V}\left(s_{1}+s_{2}\right)=\psi_{1}\left(s_{1}\right) \psi_{2}\left(s_{2}\right)\right\}$. Then $C$ is a Borel subset of $\mathbb{R}^{2}$. Since $\tilde{h}>0$ on a set of positive $\lambda_{1} * \lambda_{2}$-measure, $\lambda_{1} \otimes \lambda_{2}(C)>0$. There exist Borel subsets $A$ and $B$ of $\mathbb{R}$ such that

$$
\lambda_{1} \otimes \lambda_{2}((A \times B) \cap C) \geq \frac{3}{4} \lambda_{1}(A) \lambda_{2}(B)>0 .
$$

Given $s \in B$ define

$$
A_{s}=\left\{s_{1} \in A:\left(s_{1}, s\right) \in C\right\}
$$

and let

$$
B^{\prime}=\left\{s \in B: \lambda_{1}\left(A_{s}\right)>\frac{1}{2} \lambda_{1}(A)\right\} .
$$


In view of (15), $\lambda_{2}\left(B^{\prime}\right)>0$. If we fix $s_{2} \in B^{\prime}$, the equality $\phi_{V}\left(s_{1}+s_{2}\right)=$ $\psi_{1}\left(s_{1}\right) \psi_{2}\left(s_{2}\right)$ is satisfied for all $s_{1} \in A_{s_{2}}$. But $\lambda_{1}\left(A_{s_{2}}\right)>0$, so $A_{s_{2}}$ is uncountable, and since $\phi_{V}$ is analytic and not identically zero, we must have $\psi_{2}\left(s_{2}\right) \neq 0$. We have shown that $\psi_{2}\left(s_{2}\right) \neq 0$ for $s_{2} \in B^{\prime}$.

Now fix $s_{2}=s^{\prime}-s^{\prime \prime}$ with $s^{\prime}$ and $s^{\prime \prime}$ in $B^{\prime}$. For $s_{1} \in A_{s^{\prime}} \cap A_{s^{\prime \prime}}$, we obtain

$$
\frac{\phi_{V}\left(s_{1}+s^{\prime}\right)}{\psi_{2}\left(s^{\prime}\right)}=\frac{\phi_{V}\left(s_{1}+s^{\prime \prime}\right)}{\psi_{2}\left(s^{\prime \prime}\right)} \text {. }
$$

But $\lambda_{1}\left(A_{s^{\prime}} \cap A_{s^{\prime \prime}}\right)>0$ from the definition of $B^{\prime}$, and in particular $A_{s^{\prime}} \cap A_{s^{\prime \prime}}$ is uncountable. Both functions in (16) being analytic functions of $s_{1}$, the equality holds for all $s_{1} \in \mathbb{R}$. Now replacing $s_{1}$ by $s_{1}-s^{\prime \prime}$, we obtain that

$$
\phi_{V}\left(s_{1}+s_{2}\right)=\phi_{V}\left(s_{1}\right) \frac{\psi_{2}\left(s^{\prime}\right)}{\psi_{2}\left(s^{\prime \prime}\right)}
$$

for every $s_{1} \in \mathbb{R}$, and in particular, by taking $s_{1}=0, \phi_{V}\left(s_{2}\right)=\phi_{V}(0) \frac{\psi_{2}\left(s^{\prime}\right)}{\psi_{2}\left(s^{\prime \prime}\right)}$. But the set $B^{\prime}-B^{\prime}$ is still uncountable, so we must have $\phi_{V}(0) \neq 0$. It now follows from (17) that

$$
\phi_{V}\left(s_{1}+s_{2}\right)=\frac{\phi_{V}\left(s_{1}\right) \phi_{V}\left(s_{2}\right)}{\phi_{V}(0)},
$$

which holds for $s_{2} \in B^{\prime}-B^{\prime}$ and all $s_{1} \in \mathbb{R}$. Once $s_{1}$ is fixed, the number of $s_{2}$ being uncountable, we obtain that this equality holds for all $\left(s_{1}, s_{2}\right) \in \mathbb{R}^{2}$. We conclude that $\frac{\phi_{V}}{\phi_{V}(0)}$ is a continuous character of $\mathbb{R}$ and the proof is complete.

In order to obtain natural examples of flows satisfying the assumptions of Proposition [10 let us first notice that if $P$ is a probability Borel measure on $\mathbb{R}$ and we have the weak convergence

$$
T_{t_{n}} \rightarrow \int_{\mathbb{R}} T_{t} d P(t)
$$

of Markov operators in $L^{2}(X, \mathcal{B}, \mu)$, then

$$
e^{2 \pi i t_{n} \cdot} \rightarrow \widehat{P}(\cdot) \text { weakly-* in } L^{\infty}(\mathbb{R}, \sigma)
$$

(see e.g. the proof of Cor. 5.2 in [7]). Now, for example, if $P$ has a compact support and is not a Dirac measure or if $P$ is a Gaussian distribution, then $\widehat{P}$ is an analytic function different from a multiple of a character.

This is satisfied for many examples of special flows over irrational rotations; see e.g. [4, 6], 7], 20, 23], 24], 37].

Added in October 2009: It is proved in 21] that there are CS systems which are not DS. In fact, in 21] it is proved that a typical automorphism $T$ has a non-trivial relatively weakly mixing extension $\widehat{T}$ such that $\widehat{T}$ has the CS property.

\section{REFERENCES}

[1] O.N. Ageev, On the spectrum of Cartesian powers of classical automorphisms (Russian), Mat. Zametki 68 (2000), 643-647; translation in Math. Notes 68 (2000), 547-551. MR 1835446 (2001m:37014)

[2] O.N. Ageev, Mixing with staircase multiplicity functions, Ergodic Theory Dynam. Systems 28 (2008), 1687-1700. MR2465595 (2010c:37008)

[3] I.P. Cornfeld, S.V. Fomin, Ya.G. Sinai, Ergodic Theory, Springer-Verlag, New York, 1982. MR832433 (87f:28019) 
[4] B. Fayad, A. Windsor, A dichotomy between discrete and continuous spectrum for a class of special flows over rotations, J. Modern Dynam. 1 (2007), 107-122. MR.2261074|(2008c:37011)

[5] Y. Derriennic, K. Frączek, M. Lemańczyk, F. Parreau, Ergodic automorphisms whose weak closure of off-diagonal measures consists of ergodic self-joinings, Coll. Math. 110 (2008), 81-115. MR 2353900 (2008j:37001)

[6] K. Frączek, M. Lemańczyk, On symmetric logarithm and some old examples in smooth ergodic theory, Fund. Math. 180 (2003), 241-255. MR.2063628 (2005d:37043)

[7] K. Frączek, M. Lemańczyk, A class of special flows over irrational rotations which is disjoint from mixing flows, Ergodic Theory Dynam. Systems 24 (2004), 1083-1095. MR2085391 (2005m:37005)

[8] K. Frączek, M. Lemańczyk, On mild mixing of special flows over irrational rotations under piecewise smooth maps, Ergodic Theory Dynam. Syst. 26 (2006), 719-738. MR2237466 (2007f:37005)

[9] H. Furstenberg, Disjointness in ergodic theory, minimal sets and diophantine approximation, Math. Syst. Theory 1 (1967), 1-49. MR0213508 (35:4369)

[10] H. Furstenberg, Recurrence in Ergodic Theory and Combinatorial Number Theory, Princeton Univ. Press, 1981. MR603625 (82j:28010)

[11] E. Glasner, Ergodic Theory via Joinings, Math. Surveys and Monographs, vol. 101, Amer. Math. Soc., 2003. MR.1958753 (2004c:37011)

[12] B. Host, Mixing of all orders and pairwise independent joinings of systems with singular spectrum, Israel J. Math. 76 (1991), 289-298. MR.1177346 (93k:28022)

[13] B. Host, F. Méla, F. Parreau, Analyse Harmoniques des Mesures, Astérisque 135-136, 1986. MR839692 (88a:43005)

[14] A. del Junco, M. Lemańczyk, Generic spectral properties of measure-preserving maps, and applications, Proc. Amer. Math. Soc. 115 (1992), 725-736. MR 1079889 (92i:28017)

[15] A. del Junco, M. Lemańczyk, Simple systems are disjoint from Gaussian systems, Studia Math. 133 (1999), 249-256. MR 1687223 (2000f:28020)

[16] A. del Junco, M. Lemańczyk, Joinings of distally simple systems, preprint (2005).

[17] A. del Junco, M. Rahe, L. Swanson, Chacon's automorphism has minimal self-joinings, J. Analyse Math. 37 (1980), 276-284. MR583640 (81j:28027)

[18] A. del Junco, D. Rudolph, On ergodic actions whose self-joinings are graphs, Ergodic Theory Dynam. Systems 7 (1987), 531-551. MR922364 (89e:28029)

[19] A. del Junco, D. Rudolph, A rank-one, rigid, simple, prime map, Ergodic Theory Dynam. Systems 7 (1987), 229-247. MR896795 (88h:28016)

[20] A. Katok, Combinatorial constructions in ergodic theory and dynamics, Amer. Math. Soc., Providence, RI, 2003. MR2008435 (2004m:37005)

[21] A. Katok, M. Lemańczyk, Some new cases of realization of spectral multiplicity function for ergodic transformations, Fundamenta Math. 206 (2009), 185-215.

[22] J. King, J.-P. Thouvenot, A canonical structure theorem for finite joining-rank maps, J. Analyse Math. 56 (1991), 211-230. MR1243104 (94i:28013)

[23] M. Lemańczyk, F. Parreau, Special flows over irrational rotations with the simple convolution property, preprint (2007).

[24] M. Lemańczyk, M. Wysokińska, On analytic flows on the torus which are disjoint from systems of probabilistic origin, Fund. Math. 195 (2007), 97-127. MR2320765 (2008i:37010)

[25] F. Parreau, E. Roy, Joinings of Poisson suspensions, preprint (2007).

[26] A.A. Prikhodko, V.V. Ryzhikov, Disjointness of the convolutions for Chacon's automorphism. Dedicated to the memory of Anzelm Iwanik, Colloq. Math. 84/85 (2000), 67-74. MR:1778840(2001i:37007)

[27] M. Ratner, Horocycle flows, joinings and rigidity of products, Annals of Math. (2) 118 (1983), 277-313. MR717825 (85k:58063)

[28] J. Rosiński, T. Zak, The equivalence of ergodicity and weak mixing for infinitely divisible processes, J. Theoret. Probab. 10 (1997), 73-86. MR.1432616 (97m:60044)

[29] E. Roy, Ergodic properties of Poissonian ID processes, Ann. Probab. 23 (2007), 1163-1187. MR2308588 (2008d:60054)

[30] E. Roy, Mesures de Poisson, infinie divisibilité et propriétés ergodiques, thesis, 2005, Univ. of Paris VI. http://www.math.univ-paris13.fr/ roy/TheseRoy.pdf

[31] V.V. Ryzhikov, Joinings, wreath products, factors, and mixing properties of dynamical systems, Russian Acad. Sci. Izv. Math. 42 (1994), 91-114. MR1220583 (94h:28016) 
[32] V.V. Ryzhikov, Weak limits of powers, the simple spectrum of symmetric products, and mixing constructions of rank 1, Mat. Sb. 198 (2007), 137-159; translation in Sb. Math. 198 (2007), 733-754. MR2354530 (2008m:37008)

[33] V.V. Ryzhikov, J.-P. Thouvenot, Disjointness, divisibility, and quasi-simplicity of measurepreserving actions, Functional Analysis and its Appl. 40 - 3 (2006), 237-240. MR 2265691 (2007i:37005)

[34] J.-P. Thouvenot, Some properties and applications of joinings in ergodic theory, in: Ergodic Theory and Its Connections with Harmonic Analysis, London Math. Soc., Lecture Notes Ser. 205, Cambridge Univ. Press, 1995, 207-235. MR1325699 (96d:28017)

[35] J.-P. Thouvenot, Les systèmes simples sont disjoints de ceux qui sont infiniment divisibles et plongeables dans un flot. Dedicated to the memory of Anzelm Iwanik, Coll. Math. 84-85 (2000), 481-483. MR1784209 (2001i:28022)

[36] W.A. Veech, A criterion for a process to be prime, Monatsh. Math. 94 (1982), 335-341. MR685378 (84d:28026)

[37] D. Volný, Constructions of smooth and analytic cocycles over irrational circle rotations, Comment. Math. Univ. Carolinae 36, 4 (1995), 745 - 764. MR1378696 (97j:28041)

Faculty of Mathematics and Computer Science, Nicolaus Copernicus University, Ul. Chopina 12/18, 87-100 Toruń, Poland

E-mail address: mlem@mat.uni.torun.pl

Laboratoire d'Analyse, Géométrie et Applications, UMR 7539 Université Paris 13 et CNRS, 99 AV. J.-B. Clément, 93430 Villetaneuse, France

E-mail address: parreau@math.univ-paris13.fr

Laboratoire d'Analyse, Géométrie et Applications, UMR 7539 Université Paris 13 et CNRS, 99 AV. J.-B. Clément, 93430 Villetaneuse, France

E-mail address: roy@math.univ-paris13.fr 